\title{
Can the PTV Volume Be Used to Predict the Better Solution in Manual and Automatic Planning Using the Pinnacle Treatment Planning System for Lung Stereotactic Body Radiation Therapy?
}

\author{
Yanhua Duan \\ Shanghai Chest Hospital: Shanghai Jiao Tong University Affiliated Chest Hospital \\ Yan Shao \\ Shanghai Chest Hospital: Shanghai Jiao Tong University Affiliated Chest Hospital \\ Hua Chen \\ Shanghai Chest Hospital: Shanghai Jiao Tong University Affiliated Chest Hospital \\ Hao Wang \\ Shanghai Chest Hospital: Shanghai Jiao Tong University Affiliated Chest Hospital

\section{Hengle Gu} \\ Shanghai Chest Hospital: Shanghai Jiao Tong University Affiliated Chest Hospital \\ Aihui Feng \\ Shanghai Chest Hospital: Shanghai Jiao Tong University Affiliated Chest Hospital \\ Ying Huang \\ Shanghai Chest Hospital: Shanghai Jiao Tong University Affiliated Chest Hospital

\section{Yang Lin} \\ Shanghai Chest Hospital: Shanghai Jiao Tong University Affiliated Chest Hospital

\section{Qing Kong} \\ Fudan University
}

\section{Zhiyong Xu ( $\nabla$ xzyong12vip@sina.com )}

Shanghai Jiao Tong University Affiliated Chest Hospital https://orcid.org/0000-0002-9513-8496

\section{Research}

Keywords: Lung cancer, SBRT, PTV volume, Automatic plan, Manual plan, Cut-off point

Posted Date: September 16th, 2021

DOI: https://doi.org/10.21203/rs.3.rs-882658/v1

License: (c) (i) This work is licensed under a Creative Commons Attribution 4.0 International License. Read Full License 


\section{Abstract}

Purpose: The plan quality of the stereotactic body radiation therapy (SBRT) plan is affected by the patient's planning target volume (PTV). The predictability of PTV volume and cut-off points were investigated to judge the suitability of manual and automatic plans for lung SBRT patients.

Methods: The manual and automatic SBRT plans were retrospectively designed using the Pinnacle 16.2 treatment planning system (TPS) for 98 lung cancer patients. the suitability of manual and automatic plans for each patient is comprehensively evaluated. Receiver operating characteristic (ROC) analysis was used to investigate the predictability of PTV volume and determine the cut-off point. Once the cut-off point exists, all patients were divided into two groups according to this cut-off point. The Wilcoxon signed-rank test was performed for the dosimetric comparisons between the two groups.

Results: ROC analysis showed that PTV volume (AUC [Area under curve]: $0.918, p=0.005$ ) has diagnostic power to predict the suitability of manual and automatic plans for lung SBRT patients. The cut-off points of $22.675 \mathrm{cc}$ were selected for PTV volume. Regardless of some comparable results, the $\mathrm{Cl}, \mathrm{GI}, \mathrm{V} 10$, and V20 of automatic plans were found to be better than manual plans below the cut-off points, and the manual plan is superior to the automatic plan in $\mathrm{HI}, \mathrm{Gl}$, heart d15cc, V10, V20 above the cut-off points.

Conclusion: The PTV volume of cut-off points $(22.675 \mathrm{cc})$ are predictive of the suitability of manual and automatic plan using Pinnacle TPS for lung SBRT patients. Automatic plans were recommended for patients with PTV volumes less than 22.675cc, and manual plans can be tried for patients with larger PTV volumes.

\section{Background}

About $20-30 \%$ of patients with non-small cell lung cancer (NSCLC) are diagnosed with early NSCLC. ${ }^{[1,2]}$ Though surgery is the standard treatment of NSCLC patients, clinical evidence and studies have shown that stereotactic body radiation therapy (SBRT) can achieve a similar therapeutic effect as surgery and is a major alternative therapy for NSCLC patients unsuitable or unwilling to undergo surgery. ${ }^{[3-6]}$ Also, it is one of the major local therapies for oligometastatic lung tumors. ${ }^{[7]}$ With the advantages of short treatment time, low toxicities, and non-invasiveness, more and more clinicians and patients rank SBRT as the preferred treatment choice for patients with multiple treatment options. ${ }^{[8]}$

Like traditional manual planning, the Pinnacle treatment planning system (TPS) completes manual planning by manually adjusting optimization targets and corresponding weights. Automatic planning from Philips Pinnacle treatment planning system (TPS) is implemented by automated rule implementation and reasoning (ARIR) based methods, ${ }^{[9]}$ which firstly constructs a template including many kinds of clinical protocols, such as target and organ-atrisk (OAR) prescriptions. The TPS then begins plan optimization just like a planner and finally obtains a solution based on the selected clinical protocol to avoid excessive radiation dose deposited to healthy tissue while maximizing tumor coverage. In the optimization process of Automatic planning, some supporting structures are created to improve the OAR sparing. ${ }^{[10]}$

Some studies are comparing manual planning and automatic planning based on the Pinnacle TPS. ${ }^{[11-17]}$ These studies evaluated the pros and cons of manual and automatic planning for tumors located differently, laying the foundation for doctors and dosimeters to choose appropriate planning methods. However, most of the existing studies focus on conventional radiotherapy. SBRT has different irradiation modes and dose requirements. There are few studies on the comparison of manual and automatic SBRT planning. Secondly, Radiation Therapy Oncology Group (RTOG) 0813 [18] 
and $0915^{[19]}$ both recommend different dose constraints of the SBRT plan according to the planning target volume (PTV). However, there is no research showing whether the PTV volume can be used to predict the better solution in the manual and automatic plans.

This study generated manual and automatic plans for lung SBRT patients using the Pinnacle TPS. In addition, the overall quality of the two plans is evaluated to investigate whether the PTV volume is a predictive factor for the suitable planning method and further explore the presence of the cut-off point. It is expected that the results of this study will allow dosimeters to quickly determine the appropriate planning method for lung SBRT patients before the planning process so as to improve the efficiency of planning and provide data support for individualized treatment of patients.

\section{Materials And Methods}

\section{Data collection}

Ninety-eight lung cancer patients treated in Shanghai chest hospital from May 2019 to April 2021 were retrospectively enrolled in this study. All cases had early-stage inoperable NSCLC or oligometastatic lung cancer who were consulted with at least two radiation oncologists before receiving SBRT. The prescription dose was defined depending on clinical stage, tumor size, location, and patient's physical condition. When the study began, all the patients signed informed consent and completed their radiotherapy. The study was approved by the Institutional Ethics Committee (the committee's reference Number: KS1863).

\section{Structure delineation}

Patients were scanned with a Siemens Definition AS computed tomography (CT) Scanner System (Siemens Healthcare, Erlangen, Germany) to obtain free-breathing CT and four-dimensional CT (4DCT). All targets were delineated on a MIM Maestro Station (MIM Vista Corp, Cleveland, US-OH) by experienced radiation oncologists. The GTV was defined in the 10 phases of 4DCT. 10 GTVs were merged to generate internal target volume (ITV). PTV was obtained by expanding 0.5 $\mathrm{cm}$ of ITV in three dimensions. All structures were reviewed and approved by an independent radiation oncologist before being used for planning design.

\section{Treatment Planning}

Treatment plans were planned on the averaged 4DCT using the Pinnacle treatment planning system (TPS) (V16.2, Philips Radiation Oncology Systems, Fitchburg, WI, USA) for an Edge ${ }^{\mathrm{TM}}$ linear accelerator (Varian Medical Systems, Palo Alto, CA) equipped with a high-definition HD 120 multileaf collimator (MLC) ${ }^{\text {TM }}$. HD120 MLC ${ }^{\text {TM }}$ has 120 leaves with a leaf width projected at the isocenter of $2.5 \mathrm{~mm}$ for the central $8.0 \mathrm{~cm}$ region and $5.0 \mathrm{~mm}$ for the two $7.0 \mathrm{~cm}$ peripheral regions. The planning method was similar to our previous study. ${ }^{[8,20,21]}$ All plans employed ten or more $6 \mathrm{MV}$ fields, and the angular intervals of the fields were either 15 or 20 degrees. Collimator and couch angles were adjusted according to the individual situation. The direct machine parameter optimization (DMPO) and the collapsed cone convolution (CCC) algorithms were used for plan optimization and dose calculation with a calculation resolution of $1.0 \mathrm{~mm}$, respectively. The treatment plan goals and constraints recommended in RTOG $0813^{[18]}$ and $0915^{[19]}$ were followed, where the prescription dose covers $95 \%$ of the PTV volume.

In order to achieve a sharp dose gradient, six dose-limiting shells were generated for PTV before plan optimization. [20] The generation of those shells listed in Table 1 is briefly described as follows. First, the PTV was expanded to a specific boundary $(3 \mathrm{~mm}, 5 \mathrm{~mm}, 8 \mathrm{~mm}, 15 \mathrm{~mm}, 25 \mathrm{~mm})$ to generate an intermediate structure. The intermediate structure was then subtracted from the outline to generate a shell. This step was completed by pre-written scripts. 
Table 1

Planning constraints used for the process of optimization

\begin{tabular}{|c|c|c|c|c|c|c|c|c|}
\hline \multirow[t]{2}{*}{ Structure } & \multicolumn{4}{|l|}{ Plan-Auto } & \multicolumn{4}{|l|}{ Plan-Manual } \\
\hline & Objective & Constraint & Priority & Compromise & Objective & Constraint & Weight & Constrain \\
\hline \multirow[t]{2}{*}{ PTV } & $\mathrm{D}_{\mathrm{p}}$ & - & - & - & $V_{D p}>95 \%$ & & & Selected \\
\hline & & & & & $D_{\min }>95 \% D_{p}$ & & & Selected \\
\hline $\begin{array}{l}\text { Shell }_{3 m m} \\
\left(\% \text { of } D_{p}\right)\end{array}$ & - & $\mathrm{D}_{\max }<56$ & High & Not selected & - & $\mathrm{D}_{\max }<56$ & 100 & - \\
\hline $\begin{array}{l}\text { Shell }_{5 \mathrm{~mm}} \\
\left(\% \text { of } D_{\mathrm{p}} \text { ) }\right.\end{array}$ & - & $\mathrm{D}_{\max }<50$ & High & Not selected & - & $\mathrm{D}_{\max }<50$ & 90 & - \\
\hline $\begin{array}{l}\text { Shell }_{8 m m} \\
\left(\% \text { of } D_{p}\right)\end{array}$ & - & $\mathrm{D}_{\max }<40$ & High & Not selected & - & $\mathrm{D}_{\max }<40$ & 80 & - \\
\hline $\begin{array}{l}\text { Shell }_{11 \mathrm{~mm}} \\
\left(\% \text { of } D_{p}\right)\end{array}$ & - & $\mathrm{D}_{\max }<34$ & High & Not selected & - & $\mathrm{D}_{\max }<34$ & 70 & - \\
\hline $\begin{array}{l}\text { Shell }_{15 \mathrm{~mm}} \\
\left(\% \text { of } D_{p}\right)\end{array}$ & - & $\mathrm{D}_{\max }<28$ & High & Not selected & - & $\mathrm{D}_{\max }<28$ & 60 & - \\
\hline $\begin{array}{l}\text { Shell }_{25 \mathrm{~mm}} \\
\left(\% \text { of } D_{p}\right)\end{array}$ & - & $\mathrm{D}_{\max }<18$ & High & Not selected & - & $\mathrm{D}_{\max }<18$ & 50 & - \\
\hline \multirow{3}{*}{$\begin{array}{l}\text { Total } \\
\text { Lung (\% } \\
\text { of } \\
\text { Volume) }\end{array}$} & - & $V_{5 G y}<25$ & High & Selected & - & $V_{5 G y}<25$ & 50 & - \\
\hline & & $\mathrm{V}_{10 \mathrm{~Gy}}<15$ & High & Selected & - & $\mathrm{V}_{10 \mathrm{~Gy}}<15$ & 50 & - \\
\hline & & $V_{20 G y}<6$ & High & Selected & - & $V_{20 G y}<6$ & 50 & - \\
\hline
\end{tabular}

The optimization objectives and constraints of manual and automatic planning are optimized according to standard clinical practices. The goals and constraints for initial optimization were set by a pre-set script. The details were shown in Table 1. Some other constraints of normal tissue were individually set according to the positional relationship between each patient's target and normal tissues. In the optimization process of manual planning, objective, constraint, weight, constrain were adjusted to achieve a dose distribution as good as possible. Similarly, after optimizing automatic planning, fine-tuning was allowed by adding, reducing, or changing constraints, priority, and compromise options to achieve desirable results.

\section{Plan evaluation}

The metric of plan quality is a comprehensive evaluation index (Col) that integrates conformity index (Cl), gradient index $(\mathrm{GI})$, heterogeneity Index $(\mathrm{HI})$, and monitor units $(\mathrm{MU}) . \mathrm{Cl}$ is a parameter to evaluate the similarity between the prescription isodose line and the outer contour of PTV, which characterizes the precise coverage of prescription dose to PTV. Cl ${ }^{[22]}$ was computed as $\mathrm{Cl}=\mathrm{V}_{\mathrm{T}, \mathrm{Rx}}{ }^{2} /\left(\mathrm{V}_{\mathrm{T}}{ }^{*} \mathrm{~V}_{\mathrm{Rx}}\right)$, where $\mathrm{V}_{\mathrm{T}, \mathrm{Rx}}$ is the $\mathrm{PTV}$ volume covered by prescription dose, $\mathrm{V}_{\mathrm{T}}$ is the target volume, and $\mathrm{V}_{\mathrm{Rx}}$ is the volume covered by prescription dose. $\mathrm{Cl}$ ranges from 0 to 1 , and $\mathrm{Cl}=1$ indicates the best 
conformability. GI is a metric of dose fall-off velocity around the target area, which represents the radiation dose of OARs. GI ${ }^{[23]}$ is calculated as $\mathrm{GI}=\mathrm{V}_{50 \% \mathrm{Rx}} / \mathrm{V}_{\mathrm{Rx}}$, where $\mathrm{V}_{50 \% \mathrm{Rx}}$ is the volume receiving half the prescription dose. A lower $\mathrm{GI}$ represents a faster dose fall-off in normal tissue from the target. $\mathrm{HI}$ is an index to measure the dose uniformity of the PTV, indicating the hot spots in the target. $\mathrm{HI}$ is defined as $\mathrm{HI}=\mathrm{D}_{\max } / \mathrm{D}_{\mathrm{Rx}} \square$ where $\mathrm{D}_{\max }$ corresponds to the maximum dose of the PTV volume. $\mathrm{D}_{\mathrm{Rx}}$ is the prescription dose. Lower $\mathrm{HI}$ means a more uniform radiation distribution. For SBRT, a higher dose inside the tumor may translate to enhanced clinical efficacy in treating hypoxic tumors. ${ }^{[21,24]}$ Therefore, a higher $\mathrm{HI}$ within the clinically acceptable range of the SBRT plan represents a better plan quality. MU is an index of the complexity of a plan, which is closely related to the treatment time and delivery accuracy. ${ }^{[20,21,25]}$ The smaller MU represents a shorter treatment time and higher delivery accuracy.

Col is defined as

$\mathrm{Col}=(\mathrm{Cl} * \mathrm{HI}) /\left[\mathrm{Gl} *\left(\mathrm{MU} / \mathrm{MU}_{\mathrm{ref}}\right)\right]$

In order to avoid the excessive proportion of $\mathrm{MU}$ in the formula, we normalized $\mathrm{MU}$ with reference $\mathrm{mu}\left(\mathrm{MU}_{\mathrm{ref}}\right)$, which is defined as twice the fractional prescription dose (unit cGy). Due to the higher $\mathrm{Cl}$ and $\mathrm{HI},{ }^{[21,24]}$ lower $\mathrm{GI}$ and $\mathrm{MU}$ represent better plan quality. Therefore, a larger Col indicates a better plan.

\section{Data analysis}

Statistical analyses were performed with SPSS 20.0(IBM Corp., Armonk, NY, USA). The PTV volumes of all patients were counted in this study.

Firstly, Cols obtained from the manual plan and automatic plan were categorized according to whether the manual plan was superior to the automatic plan or not. Then, receiver operating characteristics (ROC) analysis with PTV volume as a predictor of the classification results was performed to investigate the ability of PTV volume to predict the suitability of manual and automatic plans. The predictive ability of PTV volume is reflected by the AUC value in ROC analysis. When $p<0.05$, the greater the AUC value, the better the predictive ability of PTV volume.

Secondly, if PTV volume can be used to predict the suitability of manual planning and automatic planning for lung SBRT, then the cut-off point of PTV volume for predicting the suitability of automatic planning and manual planning was tried to find. The coordinates of the ROC curve were analyzed, and the Youden index was used to determine the predictable PTV volume cut-off point. The Youden Index is a frequently used summary measure of the ROC curve. It both, measures the effectiveness of a diagnostic marker and enables the selection of an optimal cut-off point for the marker. ${ }^{[26]}$ It is defined as Youden Index = sensitivity + specificity -1 , which could be practical in daily routine with a combination of high sensitivity and specificity. The sensitivity, specificity, and positive and negative predictive values were presented when a significant cut-off value was observed. If the patient's PTV volume is larger than this cut-off point, one plan is better. Otherwise, the other plan is better.

Finally, all patients were divided into two groups below and above the cut-off point, and the Wilcoxon signed-rank test was used for the dosimetric comparison and analysis of those two groups. A p-value of less than 0.05 was considered statistically significant.

\section{Results}

\section{Patient data}

A total of 98 patient datasets were used in the study. Table 2 summarizes the descriptive statistics of the data. 
Table 2

Characteristics of the enrolled patients.

\begin{tabular}{|ll|}
\hline Factor & Value or cases \\
\hline Gender & \\
\hline Female & $31(31.63 \%)$ \\
\hline Male & $67(68.37 \%)$ \\
\hline Age (years) & \\
\hline Mean (SD) & $65(11)$ \\
\hline Median (range) & $68(33-92)$ \\
\hline Type & \\
\hline Central & 18 \\
\hline Peripheral & 80 \\
\hline ITV volume(cc) & \\
\hline Mean (SD) & $13.88(9.61)$ \\
\hline Median(range) & $12.22(0.47-32.34)$ \\
\hline PTV volume(cc) & \\
\hline Mean (SD) & $43.07(25.13)$ \\
\hline Median(range) & $41.84(4.80-128.84)$ \\
\hline Dose (Gy)/Fraction & \\
\hline $60 / 8$ & 19 \\
\hline $50 / 5$ & 26 \\
\hline $50 / 4$ & 38 \\
\hline $30 / 3$ & \\
\hline $24 / 3$ & \\
\hline
\end{tabular}

\section{Determination of PTV volume cut-off point}

ROC analysis showed that PTV volume (AUC: $0.918, p=0.005$ ) had diagnostic power to predict the suitability of manual plan and automatic plan. The ROC curve is shown in Fig. 1.

After analysis, the cut-off point of $22.675 \mathrm{cc}$ was selected for the PTV volume. Table 3 shows the sensitivity and 1 specificity corresponding to the PTV volume below and above the cut-off point. The sensitivity, specificity, and positive and negative predictive data of the cut-off points are given in Table 4. When the PTV volume is smaller than $22.675 \mathrm{cc}$, the Col of the automatic plan is higher (better) than that of the manual plan. When the PTV volume is larger than $22.675 \mathrm{cc}$, the Col of manual planning is higher (better) than that of automatic planning. 
Table 3

The sensitivity and 1 - specificity corresponding to the PTV volume below and above the cut-off point

\begin{tabular}{|lll|}
\hline Positive if Greater Than or Equal To & Sensitivity & 1 - Specificity \\
\hline 17.605 & .849 & .250 \\
18.665 & .836 & .250 \\
19.605 & .822 & .250 \\
\hline 20.535 & .808 & .250 \\
\hline 21.630 & .795 & .250 \\
\hline 22.675 & .795 & .000 \\
\hline 23.625 & .781 & .000 \\
\hline 24.645 & .767 & .000 \\
\hline 25.680 & .753 & .000 \\
\hline 26.465 & .740 & .000 \\
\hline 27.500 & .726 & .000 \\
\hline
\end{tabular}

Table 4

The sensitivity, specificity, positive and negative predictive value of the cut-off point

\begin{tabular}{|ll|}
\hline & Col (PTV $<22.675 c c$, PTV $\geq 22.675 c c)$ \\
\hline Sensitivity (\%) & 79.5 \\
\hline Specificity (\%) & 100 \\
\hline Positive predictive value (\%) & 93 \\
\hline Negative predictive value (\%) & 5 \\
\hline
\end{tabular}

\section{Case Example}

Figure 2 shows the dose distribution and DVH of patients with PTV volume below and above the cut-off point (22.675cc). Visually, when PTV < 22.675cc, compared with manual planning, the intrapulmonary 10 Gy and 20 Gy doses from automatic planning are smaller, the dose lines are more conformal to the target. The hotspots are similar for the two methods. When PTV $<=22.675 \mathrm{cc}$, compared with automatic planning, the manual planning has a smaller intrapulmonary irradiation area of 10Gy and 20Gy, smaller coverage of 25Gy, and a larger maximum dose.

\section{Dosimetric comparison of manual and automatic planning below and above the cut-off point}

Although GI can indicate the irradiation dose of normal tissues for the SBRT plan, the OARs' dosimetric results for the two groups with PTV volume below and above the cut-off point are listed in Table 5 together with other metrics for an intuitive data display. 
Table 5

Dosimetric parameters with grouping PTV volume (Mean(range))

\begin{tabular}{|c|c|c|c|c|c|c|}
\hline & \multicolumn{3}{|l|}{ PTV $<22.675 \mathrm{cc}$} & \multicolumn{3}{|l|}{ PTV $>=22.675 c c$} \\
\hline & Plan-Manual & Plan-Auto & $\mathrm{P}^{\mathrm{a}}$ & Plan-Manual & Plan-Auto & $\mathrm{P}^{\mathrm{a}}$ \\
\hline Patient & \multicolumn{3}{|l|}{44} & \multicolumn{3}{|l|}{54} \\
\hline $\mathrm{Cl}$ & $0.83(0.80-0.88)$ & $0.84(0.82-0.89)$ & $0.032^{*}$ & $0.85(0.4-0.93)$ & $\begin{array}{l}0.86(0.42- \\
0.99)\end{array}$ & 0.374 \\
\hline $\mathrm{HI}$ & $1.46(1.40-1.55)$ & $1.49(1.41-1.53)$ & 0.128 & $\begin{array}{l}1.62(1.45- \\
2.18)\end{array}$ & $\begin{array}{l}1.53(1.35- \\
1.63)\end{array}$ & $0.000^{*}$ \\
\hline Gl & $6.43(5.00-8.24)$ & $5.68(4.99-6.20)$ & $0.01^{*}$ & $\begin{array}{l}4.23(3.25- \\
6.45)\end{array}$ & $\begin{array}{l}4.72(3.50- \\
7.11)\end{array}$ & $0.000^{*}$ \\
\hline MU & $\begin{array}{l}2592.00(2046- \\
2971)\end{array}$ & $\begin{array}{l}2566.57(1912- \\
3077)\end{array}$ & 0.674 & $\begin{array}{l}\text { 1774.81(854- } \\
3076)\end{array}$ & $\begin{array}{l}1786.97(864- \\
3107)\end{array}$ & 0.702 \\
\hline $\begin{array}{l}\text { Spinal Cord } \\
D_{\max }(G y)\end{array}$ & $\begin{array}{l}11.43(14.1- \\
19.58)\end{array}$ & $10.99(15-18.59)$ & 0.314 & $\begin{array}{l}13.36(11.2- \\
27.63)\end{array}$ & $\begin{array}{l}13.95(15.4- \\
35.7)\end{array}$ & $0.015^{\star}$ \\
\hline Heart $D_{15 c c}$ & $2.90(0.12-9.00)$ & $\begin{array}{l}3.73(0.12- \\
10.78)\end{array}$ & 0.058 & $\begin{array}{l}9.60(0.24- \\
35.7)\end{array}$ & $\begin{array}{l}9.82(0.24- \\
36.29)\end{array}$ & $0.022^{*}$ \\
\hline Heart $D_{\max }$ & $\begin{array}{l}5.54(1.33- \\
15.21)\end{array}$ & $\begin{array}{l}5.04(1.33- \\
18.04)\end{array}$ & 0.123 & $\begin{array}{l}21.11(0.40- \\
45.70)\end{array}$ & $\begin{array}{l}20.58(0.42- \\
37.06)\end{array}$ & 1.000 \\
\hline Lung $\mathrm{V}_{10 \mathrm{~Gy}}(\%)$ & $5.22(2.63-6.91)$ & $5.14(1.54-7.30)$ & $0.024^{*}$ & $\begin{array}{l}10.04(1.54- \\
22.58)\end{array}$ & $\begin{array}{l}10.23(1.54- \\
22.93)\end{array}$ & $0.007^{*}$ \\
\hline Lung $\mathrm{V}_{20 \mathrm{~Gy}}(\%)$ & $2.73(1.76-5.35)$ & $2.42(1.75-3.34)$ & $0.013^{*}$ & $\begin{array}{l}5.01(4.29- \\
9.90)\end{array}$ & $\begin{array}{l}5.14(4.64- \\
9.63)\end{array}$ & $0.000^{*}$ \\
\hline Col & $0.18(0.11-0.25)$ & $0.19(0.10-0.25)$ & $0.002^{*}$ & $\begin{array}{l}0.39(0.13- \\
0.92)\end{array}$ & $\begin{array}{l}0.34(0.12- \\
0.83)\end{array}$ & $0.000^{*}$ \\
\hline \multicolumn{7}{|c|}{$\begin{array}{l}V_{10 G y} \text { and } V_{20 G y} \text { : The percentage of volume of total lung excluding ITV receiving } 10 \text { Gy and } 20 \text { Gy. } D_{\text {max }} \text { : maximum } \\
\text { dose. } D_{15 c c} \text { : Dose covering } 15 c c \text { of a structure. MLD: mean lung dose. }\end{array}$} \\
\hline & & & & & & \\
\hline
\end{tabular}

It can be seen from Table 5 that when the PTV volume is smaller than $22.675 \mathrm{cc}$, the automatic plan performs better in $\mathrm{Cl}, \mathrm{Gl}, \mathrm{V}_{10 G y}, \mathrm{~V}_{20 \mathrm{~Gy}}$, and are comparable to the manual plan for the remaining parameters ( $\mathrm{Hl}, \mathrm{MU}$, Spinal Cord $\mathrm{D}_{\max }(\mathrm{Gy})$, Heart $D_{15 c c^{\prime}}$ Heart $D_{\max }$ ). Here the overall quality of the automatic plan is better than that of the manual plan. When the PTV volume is larger than $22.675 \mathrm{cc}$, the manual plan is superior to the automatic plan in $\mathrm{HI}, \mathrm{Gl}$, Heart $\mathrm{D}_{15 \mathrm{cc}}, \mathrm{V}_{10 \mathrm{~Gy}}$, $\mathrm{V}_{20 \mathrm{~Gy}}$, similar to the auto plan in other parameters $\left(\mathrm{Cl}, \mathrm{MU}\right.$, Heart $\left.\mathrm{D}_{\text {max }}\right)$. Here the overall quality of manual planning is superior to automatic planning.

Figure 3 shows the overall evaluation parameter $\mathrm{COI}$ of manual and automatic plans. Again, it can be seen more intuitively that the better-performing plans (with higher $\mathrm{Col}$ ) are automatic and manual plans below and above the cutoff point, respectively.

\section{Discussion}


Manual planning and automatic planning are two optional planning methods in Pinnacle 16.2 TPS. This study retrospectively compared the comprehensive quality of automatic planning and manual planning of 98 lung cancer patients treated with SBRT and found that the PTV volume can be used to predict the suitability of automatic planning and manual planning. Our results show that automatic planning performs better than manual planning when the PTV volume is smaller than $22.675 \mathrm{cc}$. When the PTV volume is larger than $22.675 \mathrm{cc}$, manual planning is superior to automatic planning. As far as we know, although there are many dosimetric studies on manual planning and automatic planning, few predictive studies are on the suitability of automatic planning and manual planning for lung SBRT. The results of this study can be used to assist doctors and physicists in quickly and individually select manual or automatic planning methods for lung SBRT patients.

Generally, physicists choose automatic planning or manual planning for lung cancer SBRT patients based on their experience. However, this method is highly subjective, and the accuracy of the selection depends heavily on the physicist's experience. Alternatively, the patient's final treatment plan was determined by comparing the manual plan with the automatic plan. Although this method can ensure the accuracy of plan selection, physicists need to design at least two plans, which is labor-intensive and time-consuming. This study allows physicists or dosimeters to quickly select suitable planning methods for SBRT patients according to PTV volume, which solves the problems mentioned above in clinical practice.

The PTV volumes of the enrolled patients were almost equally spaced from $4.80 \mathrm{cc}$ to $128.84 \mathrm{cc}$ so that the reliability of the results would not be reduced due to the excessive PTV interval of the cases.

This study used the comprehensive evaluation index Col that combined $\mathrm{Gl}, \mathrm{HI}, \mathrm{Cl}$, and $\mathrm{MU}$ to select the PTV volume cutoff point. GI characterizes the normal tissue dose of an SBRT plan, $\mathrm{HI}$ reflects the curative effect of hypoxic tumor cells, $[23,24]$ and $\mathrm{Cl}$ is a characterization of prescription dose properly covering PTV. MU represents the complexity, treatment time, and delivery accuracy of a plan.

We did not incorporate normal tissues into Col because GI represents the dose gradient around the target, which can reflect the dose received by normal tissues. We investigated the dosimetry of normal tissues for patients with PTV volume below and above the cut-off point and found that the overall relative performance of the two groups for the normal tissues was the same as that of GI (Table 5), which proves once again that GI is an indicator of normal tissues dose, and Col can reasonably judge the suitability of automatic and manual planning. Although the results in Table 5 show minor absolute differences in some evaluation parameters, these dose differences may have a great biological impact for a patient treat with SBRT because of a high fractional dose, ${ }^{[21,24]}$ and hence cannot be ignored. Therefore, this study for choosing a reasonable plan is necessary.

When the PTV volume is smaller than $22.675 \mathrm{cc}$, automatic planning shows advantages in conformability, dose gradient, and part of normal tissues. And when the PTV volume is greater than or equal to $22.675 \mathrm{cc}$, manual planning is more prominent in target heterogeneity, dose gradient, and some normal tissues. In short, automatic and manual plans perform better when the PTV volume is smaller and larger than $22.675 \mathrm{cc}$, respectively.

As shown in Fig. 3, the Col below the cut-off point is lower than that above the cut-off point, indicating that the Edge accelerator results in better dosimetric results for lung cancer SBRT patients with larger PTV volume. This may be due to the mechanical design. The width of the HD120 MLC ${ }^{\mathrm{Tm}}$ at the isocenter was $2.5 \mathrm{~mm}$, which determined that the larger the tumor volume, the better performance on the plan quality. ${ }^{[21]}$

Here are some limitations of this research. As a retrospective study, patients were not randomized, which resulted in a potential selection bias. In addition, all SBRT plans are designed by the latest version 16.2 of Pinnacle TPS for an Edge accelerator. Whether the study results are applicable to lung tumors treated with other techniques, other TPS, or other 
delivery devices need to be further evaluated and confirmed. It should also be pointed that the plans involved in this research were designed based on the experience of the physicists in our center. The conclusion may be different if different plan design methods are used. The impact of the PTV volume on the suitability of manual and auto plans in other centers must be re-examined using methods similar to this study.

\section{Conclusion}

PTV volume is predictive of the suitability in manual and auto plans using Pinnacle 16.2 TPS for lung SBRT patients. Patients with PTV volumes smaller than $22.675 \mathrm{cc}$ may be candidates for treatment with auto plans. For the patients with larger PTV volumes, a manual plan can be tried. The results of this study provide a fast and theoretical way for doctors and physicists to choose appropriate planning methods for lung SBRT patients.

\section{Declarations}

Ethics approval and consent to participate[The study is a retrospective study. When the study began, all selected patients signed informed consents and completed radiotherapy. Ethical standards and patients' confidentiality were ensured and in line with regulations of the local institutional review board and data safety laws. This study was approved by the Ethics Committee of Shanghai Chest Hospital (the committee's reference Number: KS1863).

Consent for publication】All authors agree to the publication of the study.

Availability of data and material: The datasets used and/or analyzed during the current study are available from the corresponding author on reasonable request.

Competing interests: We declare that we have no financial and personal relationships with other people or organizations that can inappropriately influence our work.

Funding: Interdisciplinary Program of Shanghai Jiao Tong University, Grant/Award Number: YG2019ZDB07.

Authors' contributions: DYH, YS: data collection, statistical analysis, writing and revising the manuscript. HC, HW, HLG, AHF: statistical analysis and revising the manuscript. YH,YL: patient administration, and critical revision of the manuscript. QK, ZYX: study design, critical revision of the manuscript and funds collection. All authors gave final approval of the version to be published.

Acknowledgements: The authors thank the Nurture projects for basic research of Shanghai Chest Hospital (No. 2019YNJCM05) for its financial support.

\section{References}

1. Xiao Y, Papiez L, Paulus R, et al. Dosimetric evaluation of heterogeneity corrections for RTOG 0236: Stereotactic body radiotherapy of inoperable stage I-II non-small-cell lung cancer. International Journal of Radiation Oncology* Biology* Physics, 2009, 73(4): 1235-1242.

2. Wu D, Zhu H, Tang H, et al. Clinical analysis of stereotactic body radiation therapy using extracranial gamma knife for patients with mainly bulky inoperable early stage non-small cell lung carcinoma. Radiation Oncology, 2011, 6(1): 1-7.

3. Brooks E D, Sun B, Zhao L, et al. Stereotactic ablative radiation therapy is highly safe and effective for elderly patients with early-stage non-small cell lung cancer. International Journal of Radiation Oncology* Biology* Physics, 2017, 98(4): 900-907.

Page 10/14 
4. Nyman J, Hallqvist A, Lund J A, et al. SPACE-a randomized study of SBRT vs conventional fractionated radiotherapy in medically inoperable stage I NSCLC. Radiotherapy and Oncology, 2016, 121(1): 1-8.

5. Heal C, Ding W, Lamond J, et al. Definitive treatment of early-stage non-small cell lung cancer with stereotactic ablative body radiotherapy in a community cancer center setting. Frontiers in oncology, 2015, 5: 146.

6. Chang J Y, Senan S, Paul M A, et al. Stereotactic ablative radiotherapy versus lobectomy for operable stage I nonsmall-cell lung cancer: a pooled analysis of two randomised trials. The Lancet Oncology, 2015, 16(6): 630-637.

7. Takeda A, Kunieda E, Ohashi T, et al. Stereotactic body radiotherapy (SBRT) for oligometastatic lung tumors from colorectal cancer and other primary cancers in comparison with primary lung cancer. Radiotherapy and Oncology, 2011, 101(2): 255-259.

8. Duan Y H, Gu H L, Yang X H, et al. Evaluation of IGRT-Induced Imaging Doses and Secondary Cancer Risk for SBRT Early Lung Cancer Patients In Silico Study. Technology in Cancer Research \& Treatment, 2021, 20 : 15330338211016472.

9. Janssen T M, Kusters M, Wang Y, et al. Independent knowledge-based treatment planning QA to audit Pinnacle autoplanning. Radiotherapy and Oncology, 2019, 133: 198-204.

10. Wang M, Zhang Q, Lam S, et al. A review on application of deep learning algorithms in external beam radiotherapy automated treatment planning. Frontiers in oncology, 2020, 10: 2177.

11. Quan E M, Chang J Y, Liao Z, et al. Automated volumetric modulated arc therapy treatment planning for stage III lung cancer: how does it compare with intensity-modulated radio therapy?. International Journal of Radiation Oncology* Biology* Physics, 2012, 84(1): e69-e76.

12. Krayenbuehl J, Norton I, Studer G, et al. Evaluation of an automated knowledge based treatment planning system for head and neck. Radiation Oncology, 2015, 10(1): 1-8.

13. Hansen C R, Bertelsen A, Hazell I, et al. Automatic treatment planning improves the clinical quality of head and neck cancer treatment plans. Clinical and translational radiation oncology, 2016, 1: 2-8.

14. Speer S, Klein A, Kober L, et al. Automation of radiation treatment planning. Strahlentherapie und Onkologie, 2017, 193(8): 656-665.

15. Nawa K, Haga A, Nomoto A, et al. Evaluation of a commercial automatic treatment planning system for prostate cancers. Medical Dosimetry, 2017, 42(3): 203-209.

16. Cilla S, laniro A, Romano $\mathrm{C}$, et al. Template-based automation of treatment planning in advanced radiotherapy: a comprehensive dosimetric and clinical evaluation. Scientific reports, 2020, 10(1): 1-13.

17. Vanderstraeten B, Goddeeris B, Vandecasteele K, et al. Automated instead of manual treatment planning? A plan comparison based on dose-volume statistics and clinical preference. International Journal of Radiation Oncology* Biology* Physics, 2018, 102(2): 443-450.

18. Bezjak A, Papiez L, Bradley J, et al. Radiation Therapy Oncology Group RTOG 0813 seamless Phase I/II study of stereotactic lung radiotherapy (SBRT) For early stage, centrally located, non-small cell lung cancer (NSCLC) in medically inoperable patients. Update, 2009.

19. Videtic G M M, Hu C, Singh A K, et al. A randomized phase 2 study comparing 2 stereotactic body radiation therapy schedules for medically inoperable patients with stage I peripheral non-small cell lung cancer: NRG Oncology RTOG 0915 (NCCTG N0927). International Journal of Radiation Oncology* Biology* Physics, 2015, 93(4): $757-764$.

20. Duan Y, Gan W, Wang H, et al. On the optimal number of dose-limiting shells in the SBRT auto-planning design for peripheral lung cancer. Journal of Applied Clinical Medical Physics, 2020, 21(9): 134-142.

21. Duan Y, Cao H, Wu B, et al. Dosimetric Comparison, Treatment Efficiency Estimation, and Biological Evaluation of Popular Stereotactic Radiosurgery Options in Treating Single Small Brain Metastasis. Frontiers in Oncology, 2021: 2988.

Page $11 / 14$ 
22. Van't Riet A, Mak A C A, Moerland M A, et al. A conformation number to quantify the degree of conformality in brachytherapy and external beam irradiation: application to the prostate. International Journal of Radiation Oncology* Biology* Physics, 1997, 37(3): 731-736.

23. Treuer $\mathrm{H}$, Hoevels $\mathrm{M}$, Luyken $\mathrm{K}$, et al. Intracranial stereotactic radiosurgery with an adapted linear accelerator vs. robotic radiosurgery. Strahlentherapie und Onkologie, 2015, 191(6): 470-476.

24. Cao H, Xiao Z, Zhang Y, et al. Dosimetric comparisons of different hypofractionated stereotactic radiotherapy techniques in treating intracranial tumors $>3 \mathrm{~cm}$ in longest diameter. Journal of neurosurgery, 2019, 132(4): 10241032.

25. Morris E D, Aldridge K, Ghanem A I, et al. Incorporating sensitive cardiac substructure sparing into radiation therapy planning. Journal of applied clinical medical physics, 2020, 21(11): 195-204.

26. Fluss R, Faraggi D, Reiser B. Estimation of the Youden Index and its associated cut-off point. Biometrical Journal: Journal of Mathematical Methods in Biosciences, 2005, 47(4): 458-472.

\section{Figures}

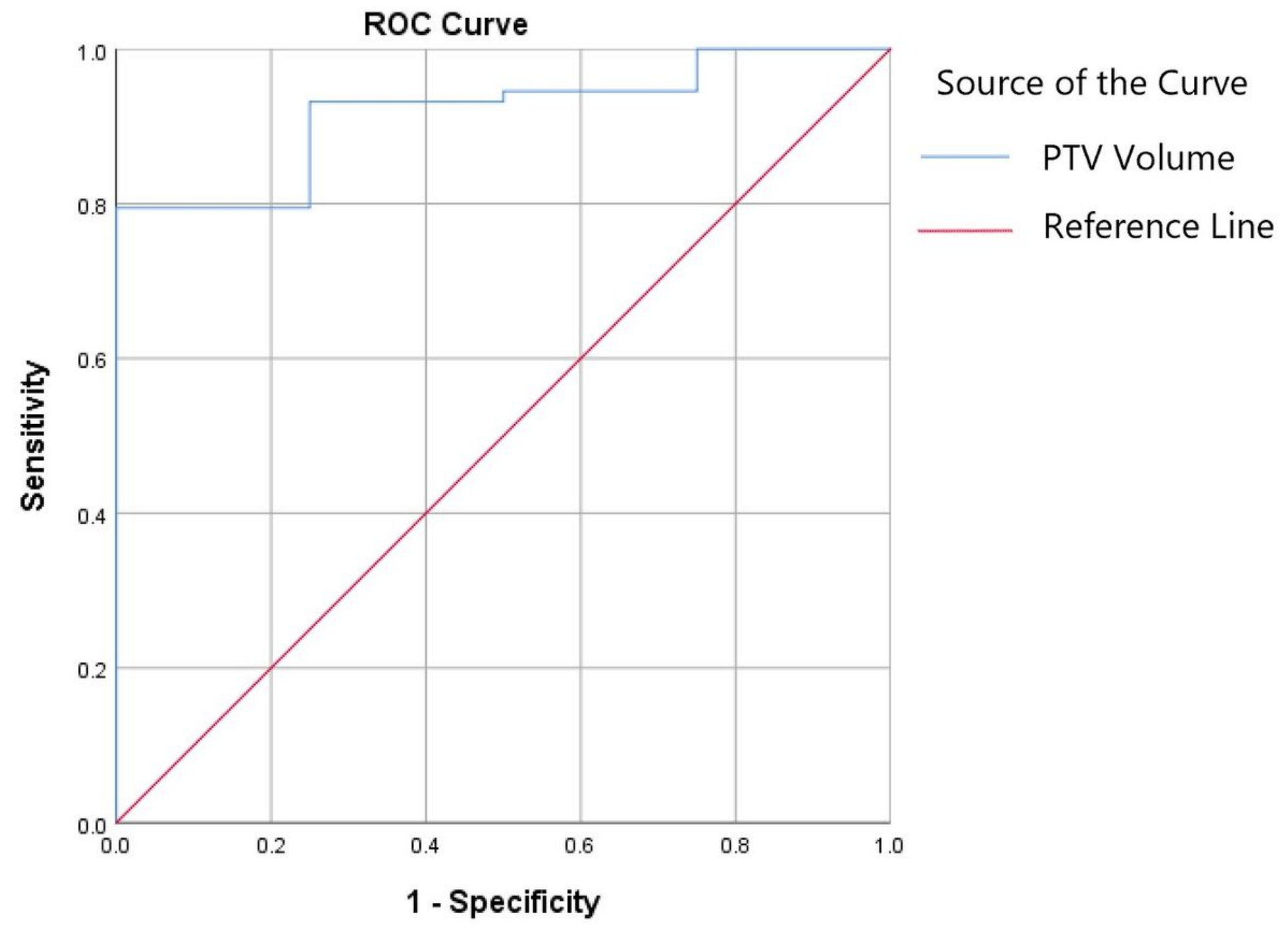

Figure 1 
PTV $<22.675$ cc PTV $>=22.675$ cC
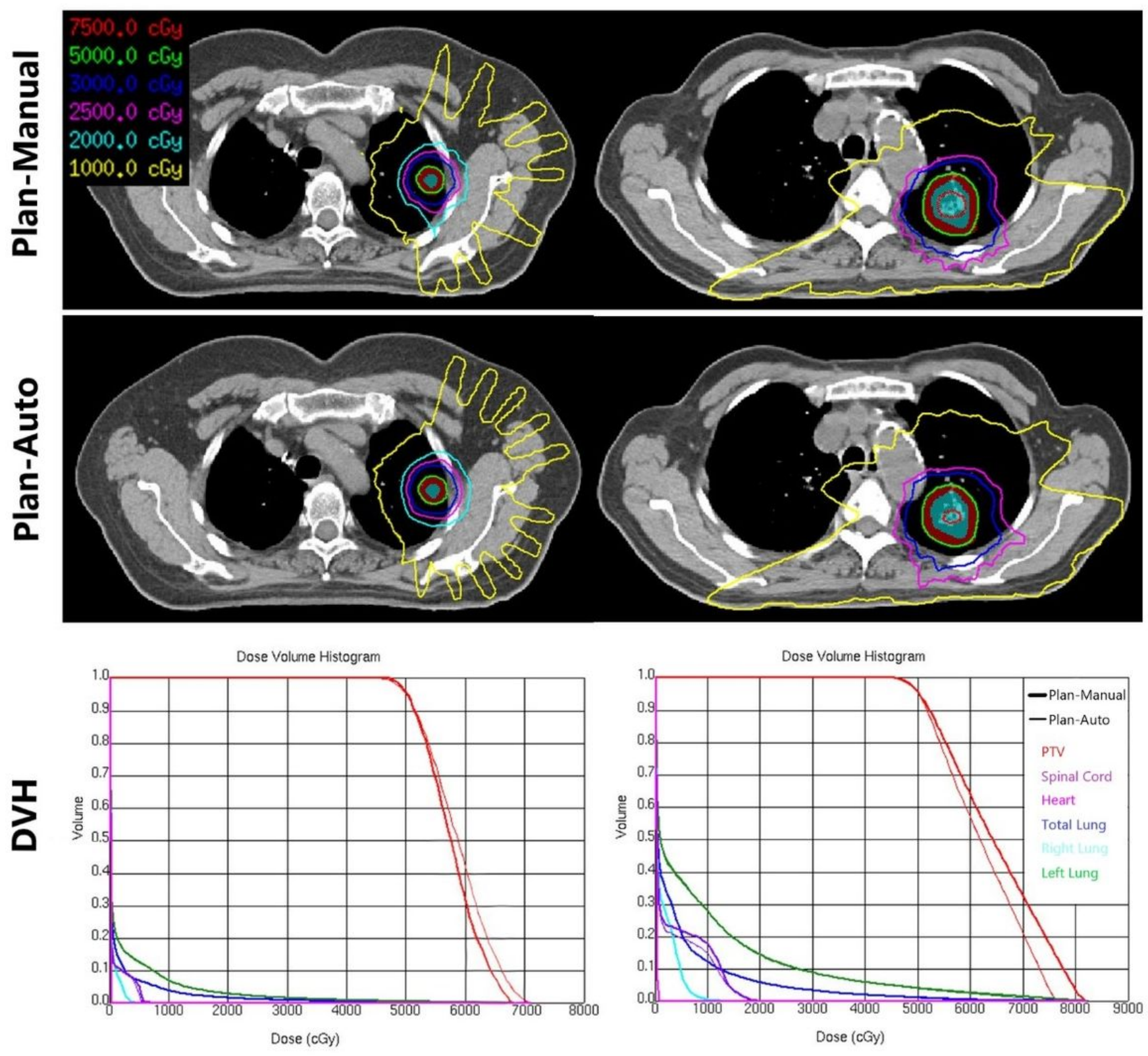

Figure 2

Dose distribution of the patients with different PTV volumes. Left: Patient with a small PTV volume. Right: Patient with a large PTV volume. Skyblue-shaded area: ITV. Red-shaded area: PTV. 


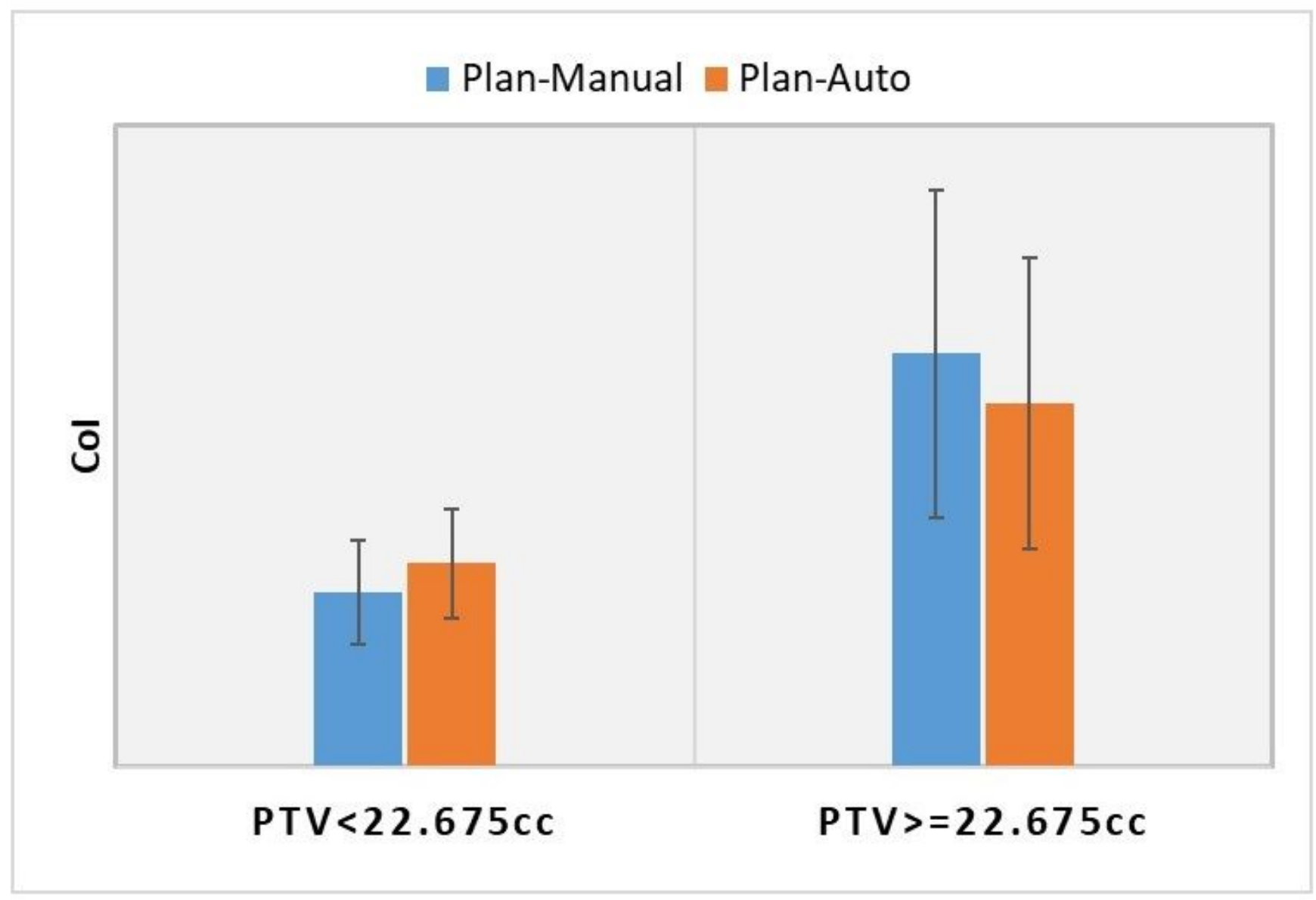

Figure 3

Overall evaluation of the automatic plan and manual plan before and after the cut-off point 